\title{
Analysis of the Linewidth of a Grating-Feedback GaAlAs Laser
}

\author{
G. Genty, A. Gröhn, H. Talvitie, M. Kaivola, and H. Ludvigsen
}

\begin{abstract}
Effects of external optical feedback from a reflection grating on the linewidth of a GaAlAs semiconductor laser operating at $780 \mathrm{~nm}$ are investigated. Accurate linewidth measurements as a function of the laser frequency tuning have been performed by applying the self-homodyne technique with a short delay line. A realistic coupled-cavity model, which incorporates the frequency-dependent reflection from the grating, is used to explain the measured data. The agreement between the theoretical and experimental results was found to be good.
\end{abstract}

Index Terms-External cavity laser, linewidth, self-homodyne method.

\section{INTRODUCTION}

D IODE lasers are known to be highly susceptible to optical feedback induced by parasitic reflections from outside the laser cavity [1], [2]. Under proper circumstances this undesirable effect can, however, also be used as an advantage. The static, dynamic, and spectral properties of the laser may be considerably influenced by coupling a portion of the diode output back into the laser cavity in a controlled fashion [3]. Such intentional optical feedback from an external reflective element allows for an effective control of, e.g., the threshold gain, the linewidth, as well as the mode stability and tuning properties of the laser [4], [5]. In practice, the behavior of a diode laser under external optical feedback can, however, be very complicated. It critically depends on the relative strength and phase of the reflected field as well as on various laser parameters. In an attempt to classify the behavior, five distinct operating regimes of external cavity diode lasers have been identified [6]. Stable single mode operation with a narrow linewidth may be obtained either with weak or strong optical feedback [7], [8]. In both categories, several device configurations have been developed over the years, and laser linewidths on the order of a few kiloHertz [9] and quasicontinuous wavelength tunings in excess of 100 $\mathrm{nm}[10]$ have been reported.

Manuscript received February 8, 2000; revised June 8, 2000. This work was supported by the Academy of Finland.

G. Genty and H. Ludvigsen are with the Metrology Research Institute, Helsinki University of Technology, FIN-02015 HUT, Finland (e-mail: goery.genty@hut.fi).

A. Gröhn was with the Metrology Research Institute, Helsinki University of Technology, FIN-02015 HUT, Finland. He is now with Nokia Research Center, FIN-00045 Nokia Group, Finland.

H. Talvitie was with the Metrology Research Institute, Helsinki University of Technology, FIN-02015 HUT, Finland. He is now with Vaisala Oyj, FIN-00421 Helsinki, Finland.

M. Kaivola is with the Materials Physics Laboratory, Helsinki University of Technology, FIN-02015 HUT, Finland.

Publisher Item Identifier S 0018-9197(00)08147-1.
Issues such as the mode stability and the influence of the threshold current on the tuning characteristics and the output power of external cavity diode lasers have been widely covered in the literature [1]-[12]. The linewidth reduction has also received attention, but to our knowledge no extensive analysis including the frequency dependence of the optical feedback and comparison with experimental data has been carried out. In this paper, we consider an external cavity diode laser under strong optical feedback from a reflection grating. We measure the linewidth variation as a function of the laser frequency detuning using the self-homodyne technique with a short delay line [13]. The experimental results are fully explained by a realistic model that includes the dispersion properties of the grating. To our best knowledge, this is the first time that such a comprehensive study combining accurate measurements with a realistic model for the laser linewidth behavior has been carried out.

The paper is organized as follows. First, we discuss in general terms the effects of optical feedback on the oscillation frequency and linewidth of an external cavity laser. This is followed by a short description of the particular laser structure studied in this work. Then we compare the measured linewidth values with those given by the applied model. Finally, we use this experimentally verified model to simulate the laser linewidth variations produced by different feedback strengths and widths of the dispersion profile of the diffraction grating.

\section{ANALYSis of OPTICAL FEEDBACK}

We consider an external cavity laser (ECL) configuration of the type depicted in Fig. 1. Here a diode laser, represented by an active medium in a short optical cavity with plane mirrors, is coupled to a much longer external cavity having a frequency selective and tunable end reflector. The amplitude reflectivities of the diode facets are $r_{1}$ and $r_{2}$ and that of the external reflector at the oscillation frequency $\omega$, including all external losses, is $r_{3}(\omega)$. In our case of strong external optical feedback the amplitude reflectivities of the diode front facet and the external reflector are related as $r_{3}>r_{2}$. The lengths of the internal diode laser cavity and the external cavity are $L_{d}$ and $L_{e}$, respectively, and the index of refraction of the active medium is $n_{d}$. The round-trip times of photons inside the internal and the external cavity are $\tau_{d}=2 n_{d} L_{d} / c$ and $\tau_{e}=2 L_{e} / c$, respectively, with $c$ being the velocity of light in vacuum.

This coupled-cavity configuration may be conveniently analyzed as a simple two-mirror laser structure by replacing the diode laser output facet reflectivity $r_{2}$ by a complex-valued effective amplitude reflection coefficient $r_{\text {eff }}$, which takes into ac- 

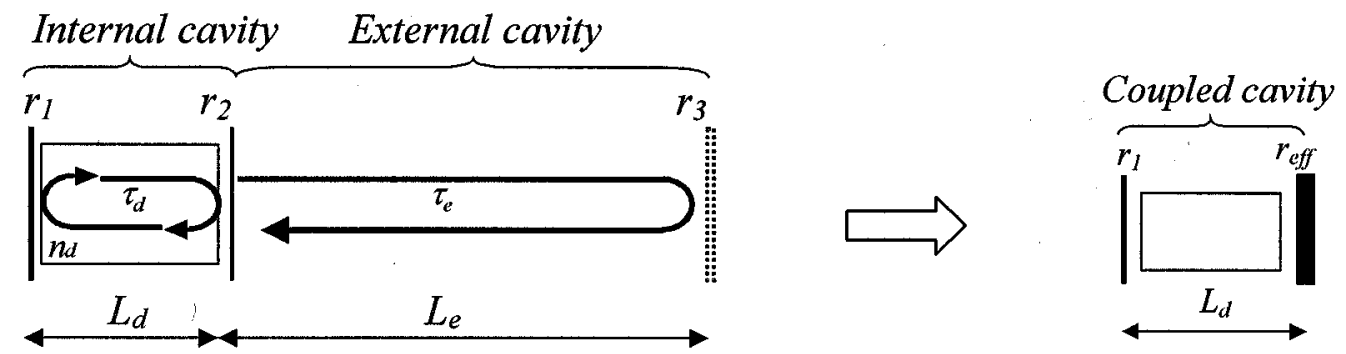

Fig. 1. Coupled-cavity model.

count the effects of both $r_{2}$ and $r_{3}(\omega)$. By keeping the $r_{2}$-surface as a reference plane $r_{\text {eff }}(\omega)$ takes the form [10]

$$
r_{\mathrm{eff}}(\omega)=\frac{r_{2}+r_{3}(\omega) e^{j \omega \tau_{e}}}{1+r_{2} r_{3}(\omega) e^{j \omega \tau_{e}}}
$$

At threshold, the condition for oscillation of the coupled-cavity laser can be expressed as [14]

$$
r_{1} e^{\left(g_{\mathrm{th}}-\alpha_{m}\right) L_{d}} e^{j \omega \tau_{d}} r_{\mathrm{eff}}(\omega)=1
$$

where $g_{\text {th }}$ is the threshold gain and $\alpha_{m}$ represents the modal loss. This gives two coupled equations from which the steadystate values of the threshold gain and the oscillation frequency of the laser may be obtained

$$
\begin{aligned}
g_{\mathrm{th}}-\alpha_{m} & =-\frac{1}{L_{d}} \ln \left(r_{1}\left|r_{\mathrm{eff}}(\omega)\right|\right) \\
\omega-\omega_{q} & =-\frac{1}{\tau_{d}} \arg \left(r_{\mathrm{eff}}(\omega)\right)
\end{aligned}
$$

with $\omega_{q}=2 \pi q / \tau_{d}$ being the frequency of the $q$ th longitudinal mode of the solitary diode laser cavity.

Above threshold, the carrier density in the semiconductor medium varies as the frequency is changed. Since the refractive index of the medium is sensitive to the carrier density, the index will vary with the frequency changes as well. To a good approximation, the dependence of the index on the threshold gain can be written as [15]

$$
n_{d}=n_{d 0}-\frac{\alpha c}{2 \omega}\left(g_{\mathrm{th}}-g_{\mathrm{tho}}\right)
$$

where $g_{\text {tho }}=\alpha_{m}-\ln \left(r_{1} r_{2}\right) / L_{d}$ and $n_{d 0}$ are the threshold gain and the refractive index of the diode laser medium in the case of no optical feedback (i.e., when $r_{3}(\omega)=0$ ). The parameter $\alpha$ is the linewidth enhancement factor given by the ratio of the real to the imaginary part of the refractive index [15], [16]. The dependence of the refractive index on the threshold gain leads to a shift in the oscillation frequency of the laser as can be noticed by combining (4) with (3b) to give

$$
\omega-\omega_{q 0}=\frac{1}{\tau_{d 0}}\left[-\arg \left(r_{\mathrm{eff}}(\omega)\right)+\alpha L_{d}\left(g_{\mathrm{th}}-g_{\mathrm{th} 0}\right)\right] .
$$

Here the subscript 0 again refers to the values of the parameters in the case of no optical feedback.
The operation characteristics of the coupled cavity laser can now be determined from (1), (3a), and (5). The variations in the frequency of the solitary diode laser and the frequency of the coupled-cavity laser are related to each other via the chirp reduction factor defined as [17]

$$
F=\frac{d \omega_{q 0}}{d \omega}=1+\frac{1}{\tau_{d 0}} \frac{d \arg \left(r_{\mathrm{eff}}(\omega)\right)}{d \omega}+\frac{\alpha}{\tau_{d 0}} \frac{d \ln \left|r_{\mathrm{eff}}(\omega)\right|}{d \omega} .
$$

According to the results of the optical transmission line model [17], the coupling to an external cavity will make the linewidth of the coupled-cavity laser $\Delta \nu$ be proportional to the inverse of the square of the chirp reduction factor [17], [18], i.e.,

$$
\Delta \nu=\frac{\Delta \nu_{0}}{F^{2}}
$$

where $\Delta \nu_{0}$ is the linewidth of the $q$ th longitudinal mode of the solitary diode laser without the optical feedback.

The coupled-cavity model presented above provides a general framework for treating the behavior of the laser linewidth in various external cavity configurations. An external cavity with a mirror as the end reflector is the simplest system for the analysis. In this case, the feedback is frequency independent, i.e., $r_{3}(\omega)$ remains constant. Moreover, since the gain profile of the GaAlAs active medium is several tens of nanometers wide [7], [19], it may be considered to be unchanged over a range of several internal cavity modes. Equations (3a) and (5) are then directly linked through the feedback phase $\omega \tau_{e}$. Fig. 2(a) shows the relation between the threshold gain and the oscillation frequency in a parametric plot obtained by letting the phase vary by $2 \pi$. The figure shows two consecutive longitudinal modes of the solitary diode laser using parameter values corresponding to the type of laser of this work (see Table I). The dotted part of the curve corresponds to unstable solutions of the laser operation for which $F<0$ [20]. Fig. 2(b) gives the corresponding behavior of the linewidth of the coupled-cavity laser with the mirror reflector as a function of the frequency detuning $\omega-\omega_{q 0}$ calculated from (5)-(7). The values presented by the dotted curves again correspond to the unstable solutions.

When a grating is used as the end reflector, the strength of the optical feedback depends on the oscillation frequency. As a consequence, the behavior of the laser linewidth as a function of the frequency detuning $\omega-\omega_{q 0}$ will change from that of 

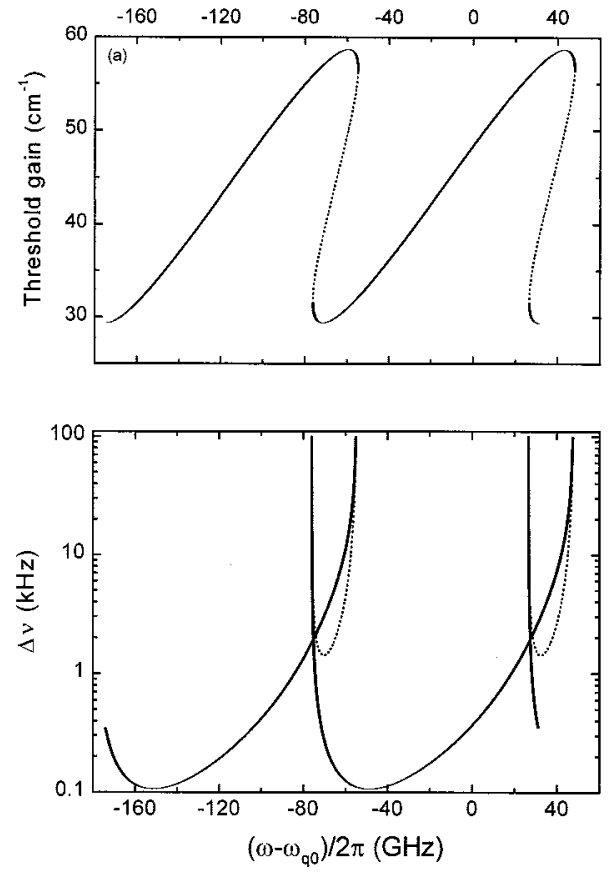

Fig. 2. Calculation of the threshold gain as a function of the detuning of the external cavity laser with a mirror as the end-reflector. The unstable regions are marked with dotted curves.

TABLE I

\begin{tabular}{ll}
\hline$r_{1}^{2}=0.95$ & $\lambda_{0}=780 \mathrm{~nm}$ \\
$r_{2}^{2}=0.05$ & $n_{d 0}=3.65$ \\
$r_{3}^{2}=0.25$ & $\alpha_{m}=20 \mathrm{~cm}^{-1}$ \\
$L_{D}=400 \mu \mathrm{m}$ & $\Delta v_{0}=20 \mathrm{MHz}$ \\
$L_{E}=12 \mathrm{~cm}$ & \\
\hline
\end{tabular}

the simple case of feedback from a mirror reflector. Assuming a Gaussian intensity profile for the beam that illuminates the grating, the reflectivity of the grating as a function of frequency will be [17]

$$
r_{3}(\omega)=r_{3} e^{-\ln 2\left(2 \frac{\omega-w_{G}}{\Delta \omega_{G}}\right)^{2}}
$$

where $\omega_{G}$ is the frequency of maximum reflection of the grating, $\Delta \omega_{G}$ the full width at half maximum (FWHM) of its spectral amplitude response, and $r_{3}$ the peak reflectivity. For each orientation of the grating, the grating dispersion selects an external cavity mode with the minimum threshold gain to oscillate within the internal cavity mode considered. To simplify the analysis, we choose $\omega_{G}$ to originally coincide with the frequency of the $q$ th longitudinal mode of the internal cavity (i.e., $\omega_{G}=\omega_{q 0}$ ). The corresponding orientation of the grating serves as a reference position. For each detuning of the spectral response curve of the grating relative to the internal cavity mode one can obtain a value for the oscillation frequency $\omega$, the chirp-reduction factor $F$, and the linewidth $\Delta \nu$ by solving the set of equations in (3a), (5), (6), and (7). When the calculation is repeated for different $\omega_{q 0}$, it results in a periodic variation of the linewidth

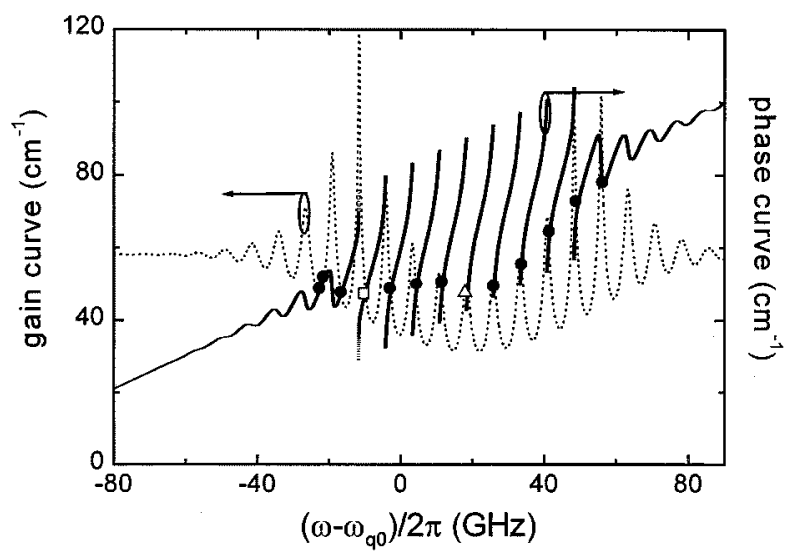

Fig. 3. Gain and phase curves as a function of the frequency detuning for $\omega_{G}-$ $\omega_{q 0}=20 \mathrm{GHz}$. For clarity, the curves correspond to a 2 -cm long external cavity. The solid circles mark possible oscillation frequencies. The rectangle gives the oscillation frequency with minimum threshold gain. The oscillation frequency corresponding to the maximum grating reflection is marked by a triangle.

as a function of the detuning with a period of $1 / \tau_{d}$. In the following, we restrict the analysis to one period, i.e., to a single internal cavity mode. To understand what happens when the grating is tuned from its original orientation, we plot the coupled equations (3a) and (5) as a function of the frequency detuning $\omega-\omega_{q 0}$. These plots are subsequently referred to as the modulus and phase curves. The solutions for the oscillation frequency are now given as the intersection points of the two curves. The curves have been plotted in Fig. 3 for a 2-cm long external cavity and a value of $\omega_{G}-\omega_{q 0}=20 \mathrm{GHz}$ for the grating detuning. The solid circles represent all the possible oscillation frequencies. The solution with minimum threshold gain, which is indicated by an open rectangle, clearly differs from the one corresponding to the maximum reflection from the grating being marked by an open triangle. An approximate assumption that these two frequencies would coincide has been made in some previous studies of this type [21].

As suggested by Fig. 3, the tuning of the grating dispersion profile relative to the frequency comb of the external cavity modes will determine the finer details of the tuning characteristics of the laser. Fig. 4 illustrates the fine-tuning of the cavity, i.e., the tuning of the lasing frequency within one external cavity mode. Part (a) of the figure shows the modulus and the phase curves for a fixed orientation of the grating. Both the oscillating external cavity mode and its neighboring mode are plotted. The effective oscillation frequency is the solution with the minimum threshold gain, which is again marked by a solid rectangle. Part (b) of the figure represents the same external cavity modes for a slightly different orientation of the grating. The shift in the grating detuning $\omega_{G}-\omega_{q 0}$ from Fig. 4(a) to (b) is equal to 1 $\mathrm{GHz}$. For small detunings (on the order of gigaHertz), the phase curve remains almost unchanged whereas the modulus curve is shifted. When the grating is rotated, the intersection point of the two curves determining the oscillation frequency sweeps the modulus curve, as indicated by the arrows in Fig. 4. Consequently, the lasing frequency of the ECL can be tuned within the external cavity modes by changing the grating orientation. Note 


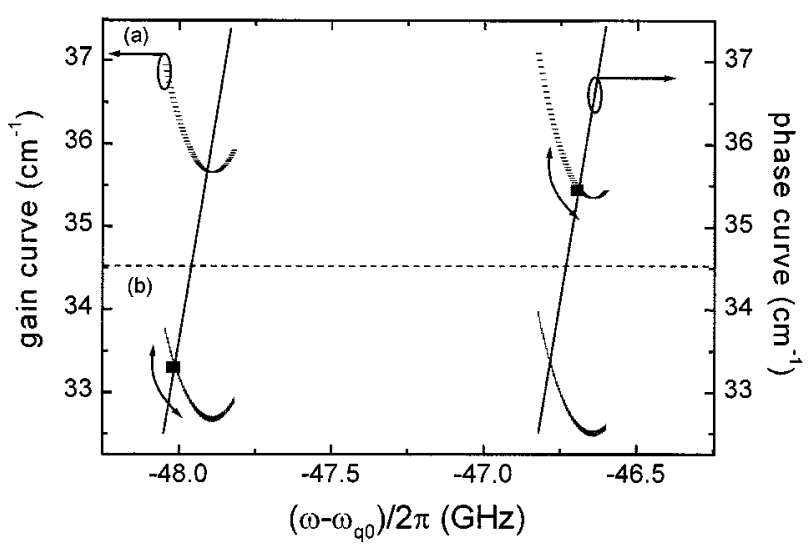

Fig. 4. (a) Gain and phase curves as a function of the frequency detuning for a particular grating position. (b) Gain and phase curves for a grating detuning increased by $1 \mathrm{GHz}$. Effective oscillation frequencies are marked by solid rectangles. Arrows show the tuning route when increasing the grating-detuning.

that the effective oscillation frequency will always be found to correspond to a crossing point on the negative slope of the gain curve.

The linewidth characteristics of the grating-cavity diode laser as a function of the grating detuning are determined by the interplay of the frequency-dependent terms in the expression of the chirp reduction factor (6). Therefore, when varying the grating angle to tune the lasing frequency of the ECL, the linewidth value is affected. Fig. 5 illustrates the behavior of the linewidth within a single internal cavity mode when changing the grating orientation. The calculated data points are marked with crosses. There is an overall trend for the linewidth to decrease with an increased detuning of the grating dispersion curve from the frequency of the solitary diode laser mode. Additionally, within each external cavity mode, the linewidth shows a variation, which is stronger for the smaller detunings and it can reach several kiloHertz. For comparison, the behavior of the linewidth of the same laser but with the grating being replaced by a simple, totally reflecting mirror is shown as a dotted line.

The amount the laser frequency can be continuously tuned within one external cavity mode by rotation of the grating depends on the length of the external cavity. For example, for the laser considered in this work with $L_{e}=12 \mathrm{~cm}$, the tuning range is only on the order of $20 \mathrm{MHz}$. This is achieved by tuning the grating by $5 \mathrm{GHz}$. On the other hand, for $L_{e}=1 \mathrm{~cm}$ the tuning range would increase to $2.5 \mathrm{GHz}$ and, correspondingly, the required tuning of the grating to $50 \mathrm{GHz}$. By combining the grating rotation with a simultaneous change of the length of the external cavity continuous frequency tuning over several external cavity mode spacings can be accomplished.

\section{EXPERIMENT AND RESULTS}

The grating-cavity laser studied in this work is schematically depicted in Fig. 6 [22]. It is composed of a GaAlAs diode laser as the active medium $\left(n_{d}=3.65\right)$, a collimating lens with a numerical aperture of 0.5 , and a focal length of $8 \mathrm{~mm}$, a beam splitter, and a gold-plated holographic diffraction grating with

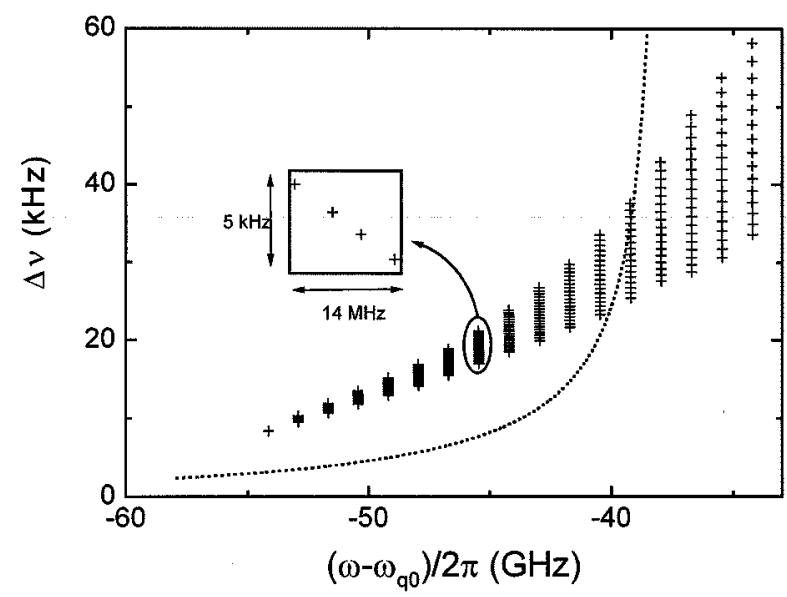

Fig. 5. Computed linewidth as a function of the frequency detuning when varying the grating orientation $\alpha=3.3$. The calculated data points are marked by crosses. The dotted line represents the linewidth computed in the case of a mirror as the end-reflector.

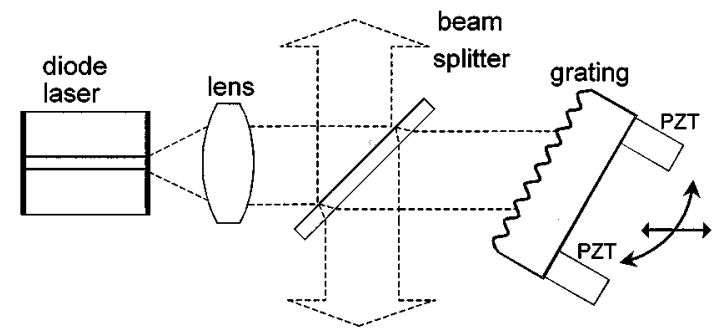

Fig. 6. Schematic layout of the grating-cavity laser of this work. The detuning of the laser is accomplished by rotating the grating with piezoelectric transducers (PZT). The beamsplitter allows us to couple out the light without changing the direction of the output beam when the laser frequency is tuned.

1800 lines $/ \mathrm{mm}$. The laser operates at $780 \mathrm{~nm}$ with a maximum output power of $40 \mathrm{~mW}$. The lengths of the internal and external cavity are $400 \mu \mathrm{m}$ and $12 \mathrm{~cm}$, respectively, corresponding to FSRs of $100 \mathrm{GHz}$ and $1.25 \mathrm{GHz}$. The intensity reflectivities of the diode laser facets are $0.95\left(r_{1}^{2}\right)$ and $0.05\left(r_{2}^{2}\right)$. The grating is mounted in the Littrow configuration. Its feedback efficiency is approximately $0.25\left(r_{3}^{2}\right)$ and the spectral width of its dispersion profile is $100 \mathrm{GHz}$. The laser can be tuned quasicontinuously over a range of $36 \mathrm{~nm}$ by rotating the grating. The beam splitter allows coupling of the laser light out from the cavity while keeping the direction of the output beam constant during the wavelength tuning.

The laser linewidth measurements were carried out using a self-homodyne technique with a delay line short compared with the coherence length of the laser. With the short delay the lowfrequency $1 / f$-noise of the laser is effectively filtered out and the measurement provides a value for the Lorentzian contribution to the laser linewidth [11] with high accuracy. The linewidth is deduced from the measurement data by fitting a theoretical spectrum based on a white noise model to the measured power spectrum using a least square fitting procedure. The accuracy of the method depends on the linewidth value and was estimated to be $\pm 1 \mathrm{kHz}$ for the range of values measured. We performed the linewidth measurement for several values of the laser detuning 


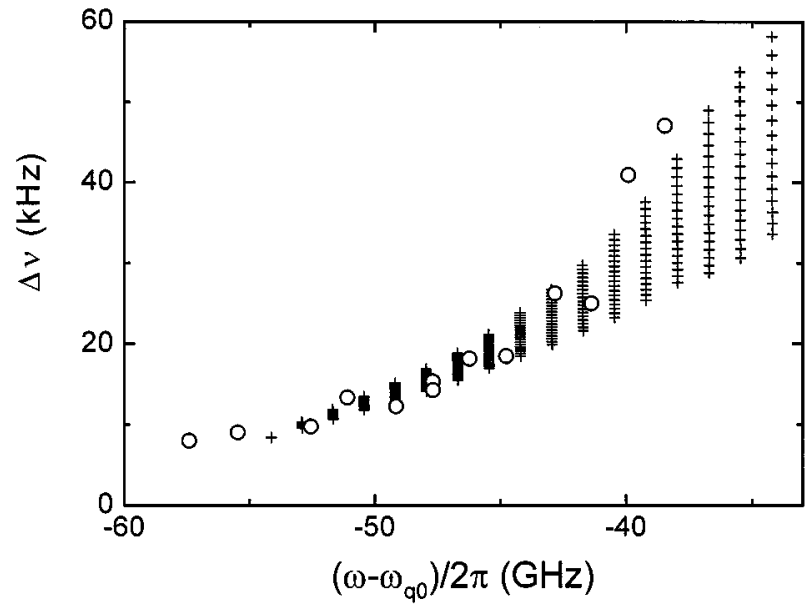

Fig. 7. Results of the laser linewidth measurements using a self-homodyne technique with a short delay. The measured data are marked by open circles. The calculated linewidth data of Fig. 5 as a function of the frequency detuning are also plotted.

$\omega-\omega_{q 0}$ within a single internal cavity mode. The detuning $\omega-\omega_{q 0}$ was determined by separately measuring the wavelength of the coupled-cavity laser and that of the diode laser in the case of no optical feedback by using an optical spectrum analyzer. The latter measurement was made by blocking the grating reflection.

The measured linewidth values vary between 8 and $48 \mathrm{kHz}$. The data are compared in Fig. 7 with the results calculated by using the model presented in the previous section. The laser linewidth depends on the $\alpha$-factor value, which is a parameter difficult to measure in practice. We repeated the calculation for several values of $\alpha$ and obtained the best agreement between theory and measurements for $\alpha=3.3$. It is important to note that the optical feedback effects the $\alpha$-factor of the laser and that the $\alpha$-factor varies with the wavelength and temperature [16]. The value for the solitary diode laser is $\alpha \cong 5$.

The overall agreement between the measured and calculated linewidth values is good. Except for the two points corresponding to the smallest detunings, the experimental data are seen to be within the range of possible values calculated for each particular detuning. This random-looking distribution of the data points is believed to be due to the fact that the relative detuning between the grating dispersion curve and the lasing external cavity mode was not controlled in the measurement when moving from one measurement point to another. We attribute the discrepancy between the simulation and the measurements for the highest linewidth values to the sensitivity of the linewidth to the $\alpha$-factor and to the grating reflectivity.

Encouraged by the good agreement between the measured and calculated linewidth values for our grating-cavity laser we use the model to predict the impact, which different feedback strengths and widths of the dispersion profile of the grating would have on the linewidth of the laser. The results are given in Figs. 8 and 9. For each external cavity mode, the plotted data points represent the average value of the calculated linewidth over the range of values obtained for the different detunings of the grating dispersion relative to the external cavity mode.

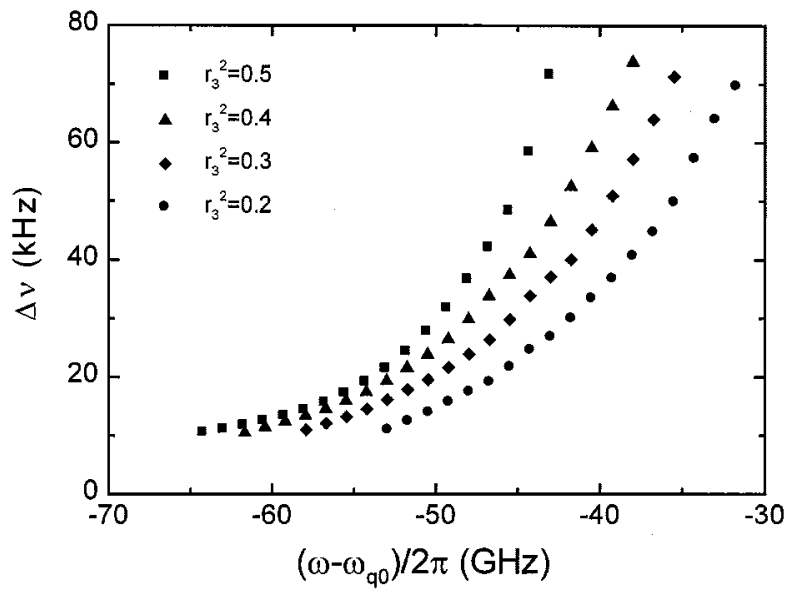

Fig. 8. Simulation of the impact of the grating diffraction efficiency on the linewidth.

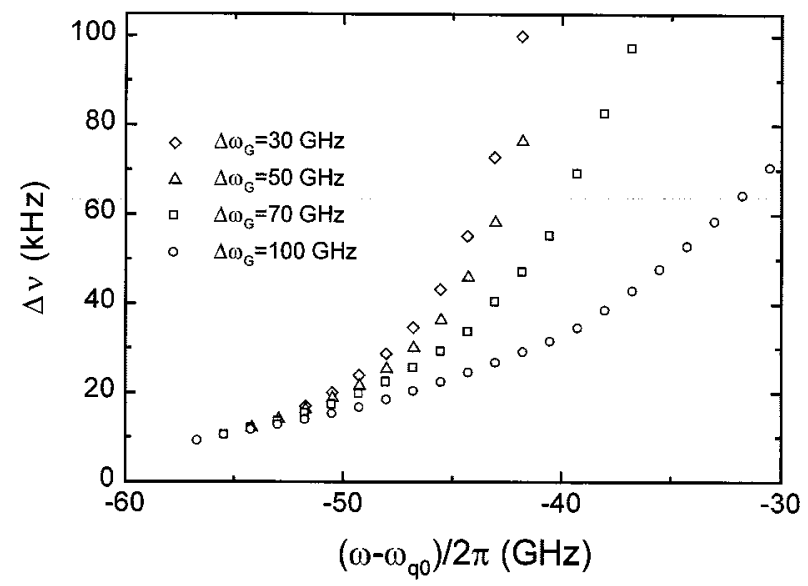

Fig. 9. Simulation of the impact of the grating width dispersion profile on the linewidth.

The impact of the strength of the grating feedback is presented in Fig. 8. The linewidth is seen to become less sensitive to the detuning from the solitary diode laser mode when the amount of feedback is increased. The tuning range within the internal cavity mode is also seen to be reduced and the frequency shift from the solitary diode laser frequency enhanced for stronger feedback. The effect of the width of the grating dispersion profile on the linewidth is illustrated in Fig. 9. The wider the profile, the larger is the range of frequency tuning within the internal cavity mode and the less sensitive the linewidth change is to the grating detuning. Moreover, the simulations show that the tuning range of the external cavity mode becomes extended for the larger widths of the grating dispersion profile. A wider range of frequencies can then be swept by the grating rotation before the laser jumps to the next internal cavity mode.

\section{SUMMARY}

We have analyzed the effects of optical feedback on the linewidth of a grating-cavity laser operating at $780 \mathrm{~nm}$. The 
laser linewidth was measured as a function of the tuning of the laser frequency using the self-homdyne detection technique with a short delay line. The experimental data were compared with the results calculated using a model, which incorporates the dispersion profile of the grating. The high accuracy of the experimental results allowed to validate the model and provided evidence on the dependency of the linewidth on the relative detuning between the grating dispersion curve and the lasing external cavity mode. The influence of the feedback strength and the width of the dispersion profile of the grating on the linewidth were investigated by simulations. These parameters were found to critically modify the tunability and the linewidth narrowing of the laser.

\section{REFERENCES}

[1] R. Lang and K. Kobayashi, "External optical feedback effects on semiconductor injection laser properties," IEEE J. Quantum Electron., vol. QE-16, pp. 347-355, 1980.

[2] K. Petermann, "External optical feedback phenomena in semiconductor lasers," IEEE J. Selected Topics Quantum Electron., vol. 1, pp. 480-489, 1995.

[3] R. Hjelme, A. R. Mickelson, and R. G. Beausoleil, "Semiconductor laser stabilization by external optical feedback," IEEE J. Quantum Electron., vol. 27, pp. 352-372, 1991.

[4] B. Tromborg, J. H. Osmundsen, and H. Olesen, "Stability analysis for a semiconductor laser in an external cavity," IEEE J. Quantum Electron., vol. 20, pp. 1023-1032, 1984.

[5] N. Schunk and K. Petermann, "Stability analysis for diodes with short external cavities," IEEE Photon. Technol. Lett., vol. 1, pp. 49-51, 1989.

[6] R. W. Tkach and A. R. Chraplyvy, "Regimes of feedback effect in 1.5- $\mu$ m distributed feedback lasers," J. Lightwave Technol., vol. LT-4, pp. 1655-1661, 1986.

[7] G. P. Agrawal, "Longitudinal-mode stabilization in semiconductor lasers with wavelength-selective feedback," J. Appl. Phys., vol. 59, pp. 3958-3961, 1986.

[8] E. Patzak, A. Sugimura, S. Saito, T. Mukai, and H. Olesen, "Semiconductor linewidth in optical feedback configurations," Electron. Lett., vol. 24, pp. 1026-1027, 1983.

[9] R. Wyatt, "Spectral linewidth of external cavity semiconductor lasers with strong, frequency-selective feedback," Electron. Lett., vol. 21, pp. $658-659,1985$

[10] H. Tabuchi and H. Ishikawa, "External grating tunable MQW laser with wide tuning range of $240 \mathrm{~nm}$," Electron. Lett., vol. 26, pp. 742-743, 1990.

[11] B. F. Skipper and H. Olesen, "Tuning characteristics of an external cavity semiconductor laser," presented at the Proceedings of IEE Colloquium: Sources for Coherent Optical Communication, London, U.K., Jan. 18, 1991.
[12] J. O. Binder, G. D. Cormack, and A. Somani, "Intermodal tuning characteristics of an InGaAsP laser with optical feedback from an external-grating reflector," IEEE J. Quantum Electron., vol. QE-26, pp. 1191-1199, 1990.

[13] H. Ludvigsen, M. Tossavainen, and M. Kaivola, "Laser linewidth measurements using self-homodyne detection with short delay," Opt. Commun., vol. 155, pp. 180-186, 1998.

[14] A. Olsson and C. L. Tang, "Coherent optical interference effects in external-cavity semiconductor lasers," IEEE J. Quantum Electron., vol. QE-17, pp. 1320-1323, 1981.

[15] C. Henry, "Theory of the linewidth of semiconductor laser," IEEE $J$. Quantum Electron., vol. QE-18, pp. 259-264, 1982.

[16] M. Osinski and J. Buus, "Linewidth broadening factor in semiconductor lasers-An overview," IEEE J. Quantum Electron., vol. QE-23, pp. 9-29, 1987.

[17] B. Tromborg, H. Olesen, X. Pan, and S. Saito, "Transmission line description of optical feedback and injection locking for Fabry-Perot and DFB lasers," IEEE J. Quantum Electron., vol. QE-23, pp. 1875-1889, 1987.

[18] R. F. Kazarinov and C. H. Henry, "The relation of line narrowing and chirp reduction resulting from the coupling of a semiconductor laser to a passive resonator," IEEE J. Quantum Electron., vol. QE-23, pp. 1401-1409, 1987.

[19] A. Asakura, K. Hagiwara, M. Iida, and K. Eda, "External cavity semiconductor laser with a Fourier grating and an aspheric lens," Appl. Opt., vol. 32, pp. 2031-2038, 1994.

[20] R. Hui and S. Tao, "Improved rate equations for external cavity semiconductor lasers," IEEE J. Quantum Electron., vol. 25, pp. 1580-1584, 1989.

[21] P. Zarobedian, W. R. Trutna, and L. S. Cutler, "Bistability in gratingtuned external-cavity semiconductor laser," IEEE J. Quantum Electron., vol. QE-23, pp. 1855-1860, 1987.

[22] H. Talvitie, A. Pietiläinen, H. Ludvigsen, and E. Ikonen, "Passive frequency and intensity stabilization of an extended-cavity diode lasers," Rev. Sci. Instrum., vol. 68, pp. 1-7, 1997.

G. Genty, photograph and biography not available at time of publication.

A. Gröhn, photograph and biography not available at time of publication.

H. Talvitie, photograph and biography not available at time of publication.

M. Kaivola, photograph and biography not available at time of publication.

H. Ludvigsen, photograph and biography not available at time of publication. 